\title{
Soft solution fluorine-free synthesis of anatase nanoparticles with tailored morphology
}

\author{
David G. Calatayud Teresa Jardiel Mónica Rodríguez, Marco Peiteado \\ Daniel Fernández-Hevia Amador C. Caballero
}

\begin{abstract}
$\mathrm{TiO}_{2}$ nanoparticles with tailored morphology have been synthesized under exceptionally soft conditions. The strategy is based on the use of a non-aqueous alcoholic reaction medium in which water traces, coming either from the air (atmospheric water) or from an ethanol-water azeotropic mixture (ethanol 96\%), are incorporated in order to accelerate hydrolysis of the Ti-precursor. Moreover, organic surfactants have been used as capping agents so as to tailor crystal growth in certain preferential directions. Combinations of oleic acid and oleylamine, which lead to the formation of another surfactant, dioleamide, are employed instead of fluorine-based compounds, thus increasing the sustainability of the process. As a result, $\mathrm{TiO}_{2}$ nanostructured hierarchical microspheres and individual nanoparticles with exposed high-energy facets can be obtained at atmospheric pressure and temperatures as low as $78{ }^{\circ} \mathrm{C}$.
\end{abstract}

\section{Introduction}

In recent years the number of published works dealing with obtaining $\mathrm{TiO}_{2}$ nanoparticles with ultra-reactive surfaces has increased exponentially, mainly due to the great interest in their high reactivity that arises both from a scientific and technological point of view. The unique physicochemical properties of $\mathrm{TiO}_{2}$ nanoparticles confer them with potential applications in a wide range of fields, acting as photocatalysts, dye-sensitive solar cells (DSCs), Li batteries, transparent conductors, gas sensors, and so on [1-13]. These properties depend not only on the crystal phase, particle size and distribution of the particles, but also on their specific morphology, since the latter can lead to a reduction in the formation of electron traps and thus facilitate the electron transfer in the semiconductor structure [1,2,14-19]. In particular, for photocatalytic applications materials based on high-energy faceted anatase nanocrystals or, as recently reported, on crystalline hierarchical $\mathrm{TiO}_{2}$ hollow spheres [20-22] are required, in order to achieve a high efficiency. Accordingly, the synthesis of well-crystallized and nanostructured $\mathrm{TiO}_{2}$ particles with tailored morphology represents a major challenge.

The formation and growth of $\mathrm{TiO}_{2}$ nanoparticles is generally achieved through hydrolysis and condensation processes from different precursors. The major problem tailoring the morphology and size of these particles arises from the titanium ions themselves: $\mathrm{Ti}^{4+}$ cations are large and highly electropositive, and therefore prone to suffer a nucleophilic attack by water, which leads to a too fast hydrolysis and thus to uncontrolled growths and precipitations. One of the most effective ways to control the rate of hydrolysis is through the choice of the metal alkoxide, e.g. altering the OR groups. The alkoxy groups which are most frequently used in the synthesis of $\mathrm{TiO}_{2}$ comprise from 2 (ethoxide) to 4 (butoxide) carbon atoms, and their 
reactivity during the hydrolysis decreases with increasing chain length. However, in the presence of excess of water, hydrolysis is still exothermic and quick and completed in seconds. A feasible way of further reducing this speed is by adjusting the $\mathrm{H}_{2} \mathrm{O} / \mathrm{M}(\mathrm{OR}) n$ ratio and stabilizing the formed colloids. What is more, the replacement of the alkoxy groups by less hydrolyzable ones may also contribute to decrease the rates of hydrolysis and condensation, affecting the crystallization behavior and increasing the control over the final particle size. Even with these procedures, the overall hydrolysis reaction of $\mathrm{Ti}^{4+}$ ions still remains too fast, and thus a very fine control of the reaction conditions is a necessary requirement, since any slight alteration of the reaction kinetics will cause dramatic changes in the size and morphology of the final material.

In order to overcome this drawback, non-hydrolytic processes have been described for obtaining $\mathrm{TiO}_{2}$ nanoparticles with high crystallinity. The use of oxygen donor groups other than water may allow a better control of the reaction rate, leading to slow particle growth. The problem is that under these conditions the reaction rate is drastically reduced, the process becomes excessively slow and the possibility of tailoring the morphology of the synthesized particles is hindered by kinetic considerations [23-27]. In this sense a promising alternative which has been recently considered combines both hydrolytic and non-hydrolytic reactions: by adding a certain amount of water into a nonaqueous media, the size and morphology of the $\mathrm{TiO}_{2}$ precipitated particles can be finely tuned [28].

However, for the particular case of high-energy faceted $\mathrm{TiO}_{2}$ nanocrystals an extra problem needs to be faced: due to minimization of the surface energy, the number of highly reactive faces rapidly decreases during the crystallization process. This means that under normal conditions the synthesized $\mathrm{TiO}_{2}$ crystals consist mainly of poorly reactive $\{101\}$ faces, which are thermodynamically more stable than the desired $\{001\}$ and $\{010\}$ faces (Fig. 1). In order to reverse this situation, i.e. to stabilize the more reactive faces, a characteristic resource is the use of morphological agents that cap the growth in one or more
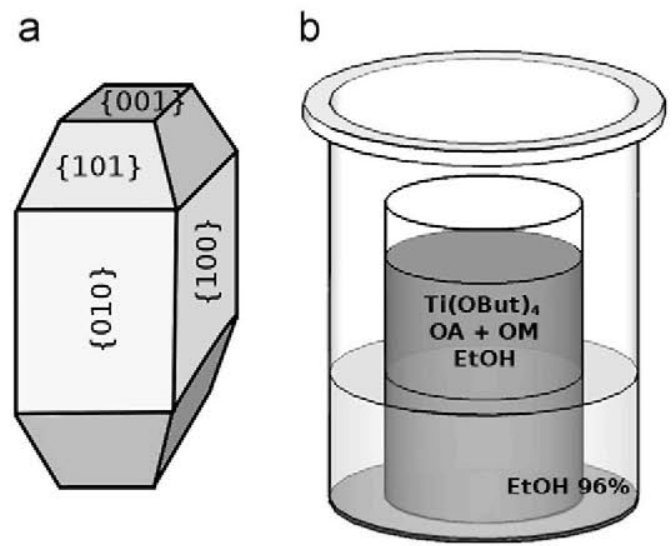

Fig. 1. (a) Anatase $\mathrm{TiO}_{2}$ with $\{101\},\{001\}$ and $\{010\} /\{100\}$ facets and (b) scheme of the reaction assembly for the nanoparticles synthesis. faces, making the crystal develop only in certain directions and hence altering/controlling its morphology [29]. Hydrofluoric acid is one of these agents, and different experimental and theoretical studies have proved its efficiency producing faceted $\mathrm{TiO}_{2}$ crystals with high percentages of $\{001\}$ and $\{010\}$ faces [30-33]. Most recent works on the synthesis of faceted $\mathrm{TiO}_{2}$ follow this methodology, in which fluorine anions selectively adsorb on the surface of $\{010\}$ and $\{001\}$ faces, stabilizing them. However, the use of fluorinated compounds as morphological control agents also carries some disadvantages: first an input of pressure and temperatures around $180^{\circ} \mathrm{C}$ are typically required so as to obtain the properly crystallized faceted particles. Moreover, during the reaction process a release of highly corrosive hydrofluoric vapors is always observed, which undoubtedly represents a major drawback for the largescale synthesis of faceted $\mathrm{TiO}_{2}$. In view of these facts, the substitution of fluorine-based compounds by organic capping surfactants which usually contain amine, amide or carboxylic groups, must be considered. Used either individually or combined [25-28,34-36], these molecules represent a more environmentally-friendly option to tune the morphology of the anatase particles. To be more precise, Dinh et al. [28] have recently proved different combinations of oleic acid and oleylamine to successfully synthesize $\mathrm{TiO}_{2}$ crystals with different shapes. The problem with fluorine vapors is consequently eradicated when using this method, but temperatures around $180^{\circ} \mathrm{C}$ and the use of a sealed autoclave reactor to autogenously increase the pressure are still required.

In this sense, herein we report a new synthetic approach based on a non-aqueous and fluorine-free technique to obtain $\mathrm{TiO}_{2}$ nanocrystals with tailored morphology at atmospheric pressure and temperature clearly below $100^{\circ} \mathrm{C}$. Nanostructured hierarchical $\mathrm{TiO}_{2}$ spheres as well as individual faceted anatase nanocrystals are successfully prepared at remarkably soft conditions. Oleic acid and oleylamine are combined and used as morphological control agents, and their capping role is analyzed and reinterpreted in terms of the formation of the dioleamide as a surfactant molecule.

\section{Materials and methods}

\subsection{Chemicals}

The chemicals titanium (IV) tetrabutoxide (Ti(OBut) $)_{4}$, Fluka, 98\%), ethanol (EtOH, Merck, analytically pure), oleic acid ((Z)-octadec-9-enoic acid, designated as OA, Fluka, analytically pure), and oleylamine ((Z)-octadec-9en-1-amine, designated as OM, Aldrich, $70 \%$ ) were used without further purification.

\subsection{Synthesis of $\mathrm{TiO}_{2}$ spheres ( $\mathrm{TiS}$ )}

A solution of $\mathrm{Ti}(\mathrm{OBut})_{4}(10 \mathrm{mmol})$ in $200 \mathrm{~mL}$ of dry ethanol was stirred under reflux for $8 \mathrm{~h}$. Then the solution 


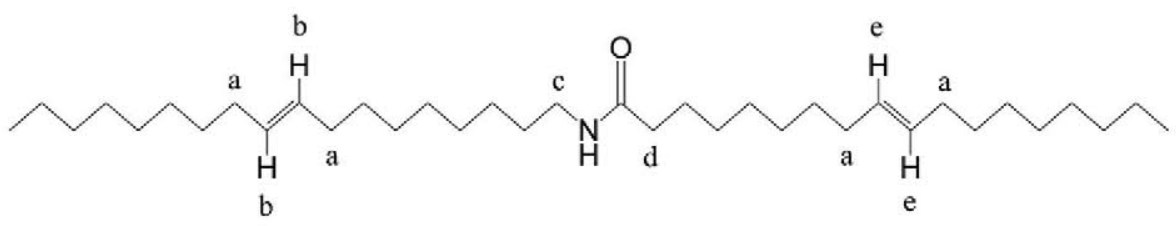

Fig. 2. Dioleamide (DO).

was evaporated to dryness, and the obtained white solid was washed with deionized water and dried at room temperature. The as-synthesized $\mathrm{TiO}_{2}$ spheres were calcined at 300 and $400{ }^{\circ} \mathrm{C}$ for $4 \mathrm{~h}$ so as to remove organic products and promote crystallinity.

\subsection{Synthesis of dioleamide (DO)}

OA $\quad(3.55 \mathrm{~g}, \quad 12.5 \mathrm{mmol})$ and $\mathrm{OM} \quad(70 \%, 4.80 \mathrm{~g}$, $12.5 \mathrm{mmol}$ ) were mixed in a flask under stirring, heat release was appreciated. The obtained yellow liquid was dried with $\mathrm{MgSO}_{4}$. Yield: $99 \% .{ }^{1} \mathrm{H}$ NMR $(300 \mathrm{MHz}$, DMSO-d $6, \delta): 7.35\left(\mathrm{~s}, 2 \mathrm{H}, \mathrm{H}_{\mathrm{b}}\right), 5.28\left(\mathrm{~m}, 3 \mathrm{H}, \mathrm{H}_{\mathrm{e}}+\mathrm{NH}\right)$, $2.66\left(\mathrm{~s}, 2 \mathrm{H}, \mathrm{H}_{\mathrm{c}}\right), 1.98\left(\mathrm{~s}, 8 \mathrm{H}, \mathrm{H}_{\mathrm{a}}\right), 1.51\left(\mathrm{~s}, 2 \mathrm{H}, \mathrm{H}_{\mathrm{d}}\right), 1.26(\mathrm{~s}$, $46 \mathrm{H}, \mathrm{CH}_{2}$ ), 0.85 (q, 6H, $\mathrm{CH}_{3}$ ) (see Fig. 2).

\subsection{Synthesis of $\mathrm{TiO}_{2}$ nanoparticles ( $\mathrm{TiNP}$ )}

In a typical procedure, $\mathrm{Ti}(\mathrm{OBut})_{4}(10 \mathrm{mmol})$ was added to a mixture of $\mathrm{X} \mathrm{mmol} \mathrm{OA}$, Y mmol OM, in $5: 5$ or $6: 4$ ratios, and $200 \mathrm{mmol}$ of dry ethanol. The Pyrex beaker containing the obtained mixture was placed into an automated laboratory reactor system (Mettler-Toledo LabMax) containing $80 \mathrm{~mL}$ of ethanol $96 \%$ (see Fig. 1). Then the reaction was heated under stirring at $78^{\circ} \mathrm{C}$ for $8 \mathrm{~h}$ or $72 \mathrm{~h}$. The obtained precipitate was washed with dry ethanol and deionized water several times and then dried at room temperature.

\subsection{Surface surfactants removal from as-prepared anatase TiNP}

Solid samples of the as-prepared anatase $\mathrm{TiO}_{2}$ nanoparticles were heated at $200{ }^{\circ} \mathrm{C}$ for $30 \mathrm{~min}$. The obtained orange solids were then washed with hot dry ethanol under stirring several times. Finally the obtained white precipitates were washed with deionized water and one last time with ethanol. The clean samples were dried at $80^{\circ} \mathrm{C}$ in air.

\subsection{Characterization}

The analyses of the crystalline structure and phase identification were performed by X-ray diffraction (XRD Bruker D8 ADVANCE) with a monochromatized source of $\mathrm{CuK}_{\alpha 1}$ radiation $(\lambda=1.5406 \mathrm{~nm})$ at $1.6 \mathrm{~kW}(40 \mathrm{kV}$, $40 \mathrm{~mA}$ ). Samples were prepared by placing a drop of a concentrated ethanolic dispersion of particles onto a silicon single crystal. FESEM images were obtained with a Hitachi S-4700 field-emission scanning electron microscope (FESEM) working at $20 \mathrm{kV}$. Transmission electron microscopy (TEM) images were collected in a HITACHI H7100 microscope working at $125 \mathrm{kV}$ and high-resolution transmission electron microscopy (HRTEM) images were obtained on a JEOL 2100F transmission electron microscope (TEM/STEM) operating at $200 \mathrm{kV}$ and equipped with a field emission electron gun, providing a point resolution of $0.19 \mathrm{~nm}$; samples were prepared by placing a drop of a dilute toluene or ethanol dispersion of nanoparticles onto a 300 mesh carbon-coated copper grid and evaporated immediately at $60{ }^{\circ} \mathrm{C}$. ${ }^{1} \mathrm{H}$ NMR spectra were recorded on a spectrometer Bruker AMX-300 using DMSO- $\mathrm{d}_{6}$ as solvent and TMS as internal reference.

\section{Results and discussion}

$\mathrm{TiO}_{2}$ particles with distinct morphologies have been synthesized under soft, non-aqueous, ethanolic conditions. All reactions were carried out at $78^{\circ} \mathrm{C}$ using $\mathrm{Ti}(\mathrm{ButO})_{4}$ as the starting reagent. $\mathrm{Ti}(\mathrm{ButO})_{4}$ was chosen as $\mathrm{Ti}$ precursor for its slow rate of hydrolysis, due to the butoxide group, which slows down the process of diffusion and polymerization compared to other alkyl groups. To obtain $\mathrm{TiO}_{2}$ nanoparticles a new synthesis method was developed from the basis of that reported by Dinh et al. [28], with EtOH $(96 \%)$ providing the water necessary to accelerate the hydrolysis reaction, and oleic acid (OA) and oleylamine $(\mathrm{OM})$ as capping agents. Experiments were run in the absence of surfactants as well as with different ratios of oleic acid and oleylamine. In those experiments involving surfactants, the $\mathrm{Ti}(\mathrm{OBut})_{4}$ : surfactant molar ratio was kept constant at 1:10.

\subsection{Growth in the absence of surfactants}

When no surfactant is used, $\mathrm{TiO}_{2}$ spheres with homogeneous sizes $(2-4 \mu \mathrm{m})$ are obtained (Fig. 3). A more detailed analysis of the surface shows that the particles are in fact aggregates of $\mathrm{TiO}_{2}$ nanocrystals with a spherical macrostructure (Fig. 3 (b), (d) and (f)), similar to those described in previous works $[21,22,37]$. This growth should be ascribed to the synthesis route: at low temperature and due to the limited amount of water from air, the formed $\mathrm{TiO}_{2}$ crystals are small, and assemble in Ti microspheres with nanosized pores [20]. The crystallinity of the synthesized samples was examined by powder X-Ray diffraction. As shown in Fig. 4, the precipitate presents a low degree of crystallinity, which might be due to the small size of the 

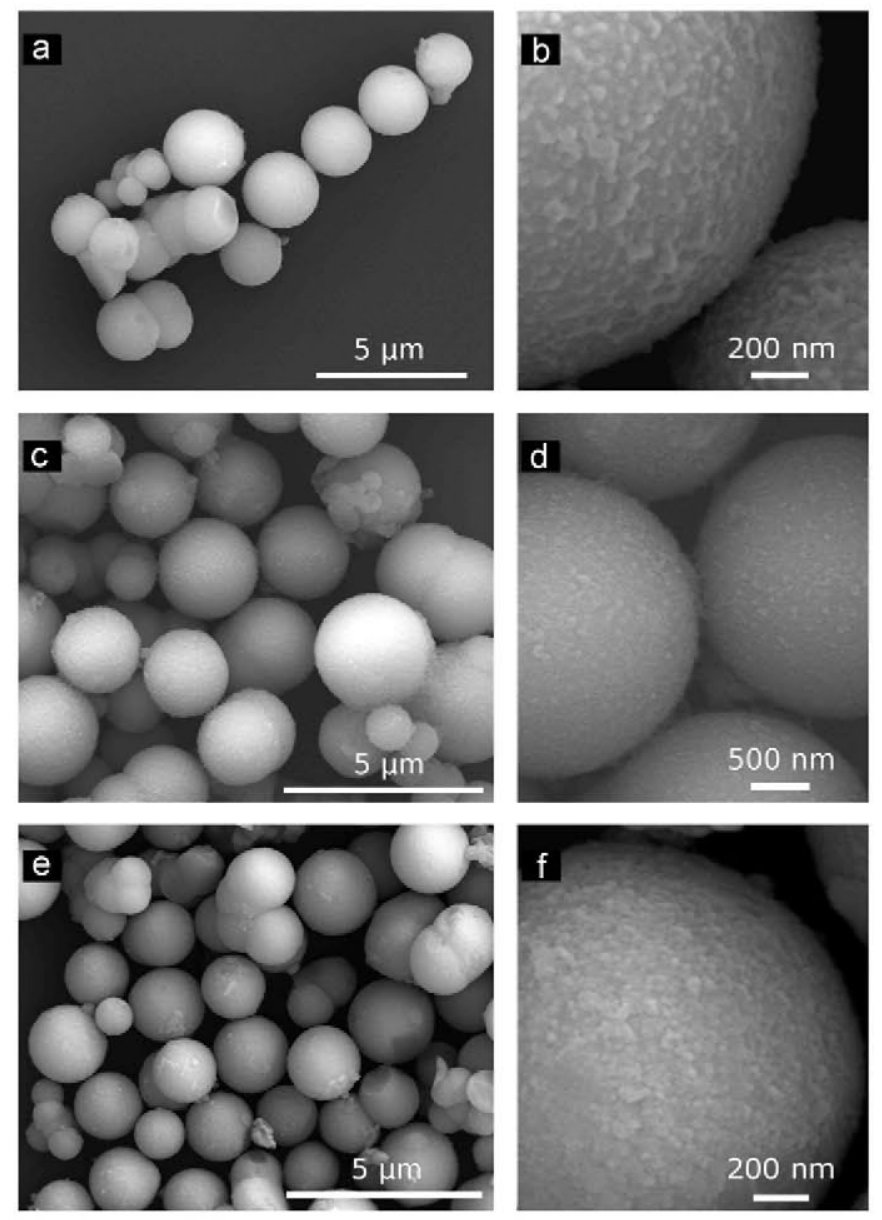

Fig. 3. (a) FESEM micrograph of the sample TiS, (b) detail of the surface of TiS, (c) FESEM micrograph of the sample TiS300, (d) detail of the surface of TiS300, (e) FESEM micrograph of the sample TiS400 and (f) detail of the surface of TiS400.

nanocrystals constituting the spheres $(\sim 5 \mathrm{~nm})$. This fact is confirmed in the TEM micrographs (see Fig. 5), whereby the $\mathrm{TiO}_{2}$ spheres, formed by small nanocrystals, show a cloud-like appearance. In order to improve the crystallinity of the obtained particles these samples were fired at temperatures of $300{ }^{\circ} \mathrm{C}$ (TiS300 samples) and $400{ }^{\circ} \mathrm{C}$ (TiS400 samples) for $4 \mathrm{~h}$. As it can be seen in Fig. 4, the degree of crystallinity increases after calcination, due to a growth and consolidation of the nanoparticles (Fig. 3). In both cases crystallization of pure anatase phase (JCPDS File no. 21-1272) is observed, and FESEM micrographs confirm that the size and macrostructure of the spheres are maintained after calcination (Fig. 3). Therefore, this method of synthesis allows us to obtain crystalline $\mathrm{TiO}_{2}$ spheres with homogeneity of sizes and shapes, similar to those described by other authors, but employing mild reaction conditions.

\subsection{Effect of surfactants and surfactants ratio}

The situation significantly changes when the reactions are carried out in the presence of oleylamine and oleic acid,

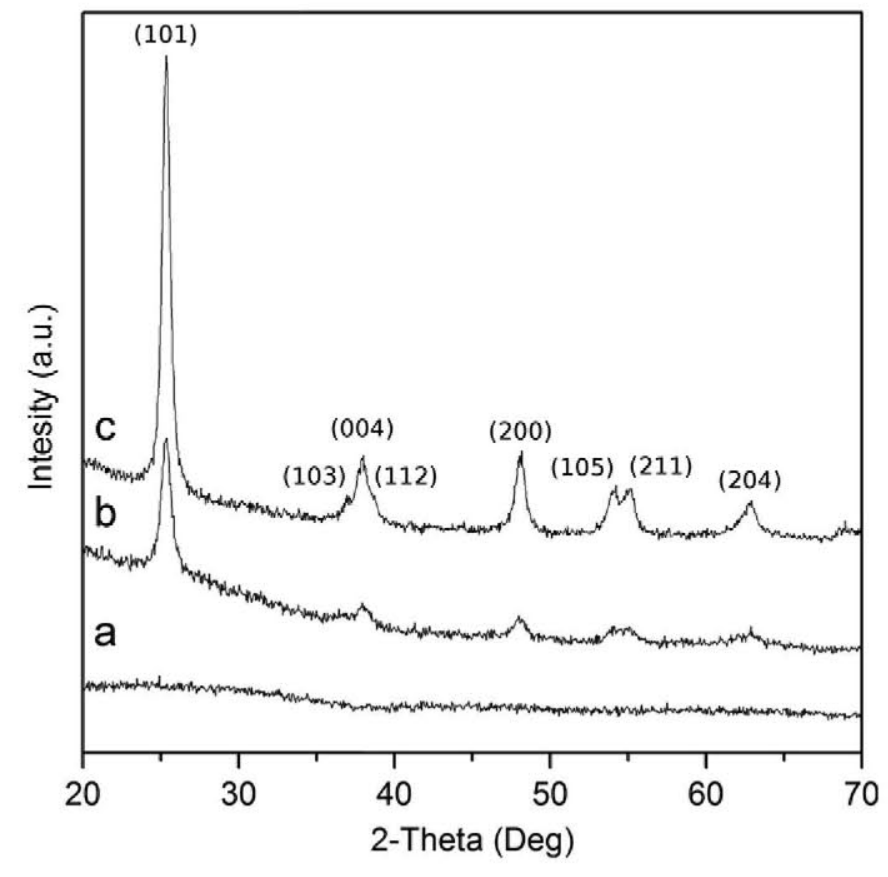

Fig. 4. XRD diffraction patterns of the TiS samples: (a) TiS, (b) TiS300 and (c) TiS400.

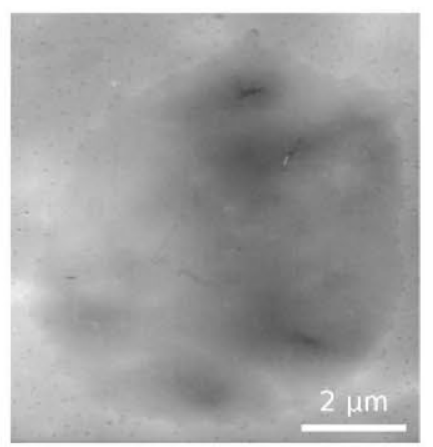

Fig. 5. Low-magnification TEM micrograph of an individual sphere of the sample TiS

since now faceted nanoparticles of $\mathrm{TiO}_{2}$ are obtained. More specifically, when the OA/OM ratio is 5:5 (TiNP5 samples), truncated rhombic-shaped $\mathrm{TiO}_{2}$ nanoparticles are obtained, with a size of about $25 \mathrm{~nm}$ (Fig. 6(a)). A modification of the surfactants ratio to 6:4 (TiNP6 samples) leads to the formation of $\mathrm{TiO}_{2}$ nanoparticles with similar size and morphology, together with the formation of $\mathrm{TiO}_{2}$ nanorods (Fig. 6(b)). The crystallinity of the synthesized samples was verified by selected area electron diffraction (SAED) (Fig. 6(c)). This change in the growth habit with regard to the surfactant-free compositions must be interpreted as follows: as mentioned in the introduction, the shape of the final products is affected by the rate of the two main processes which control the formation of $\mathrm{TiO}_{2}$, i.e. hydrolysis of the titanium precursor and the subsequent condensation reactions to form a $\mathrm{Ti}-\mathrm{O}$ - Ti network [38]. By using surfactants with different functional groups and distinct binding strengths, the morphology of resulting 

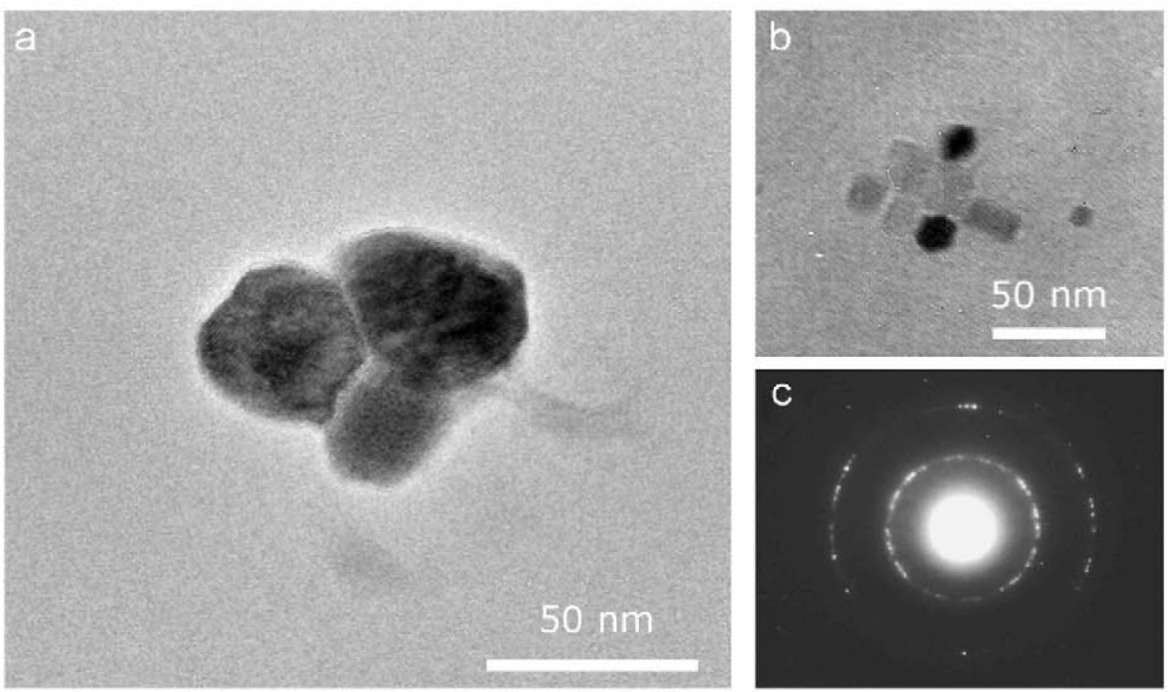

Fig. 6. Representative TEM images of: (a) TiNP5 obtained at $78{ }^{\circ} \mathrm{C}$ for $8 \mathrm{~h}$, (b) TiNP6 obtained at $78^{\circ} \mathrm{C}$ for $8 \mathrm{~h}$ and (c) SAED of truncated rhombic $\mathrm{TiO}_{2}$ nanoparticles.
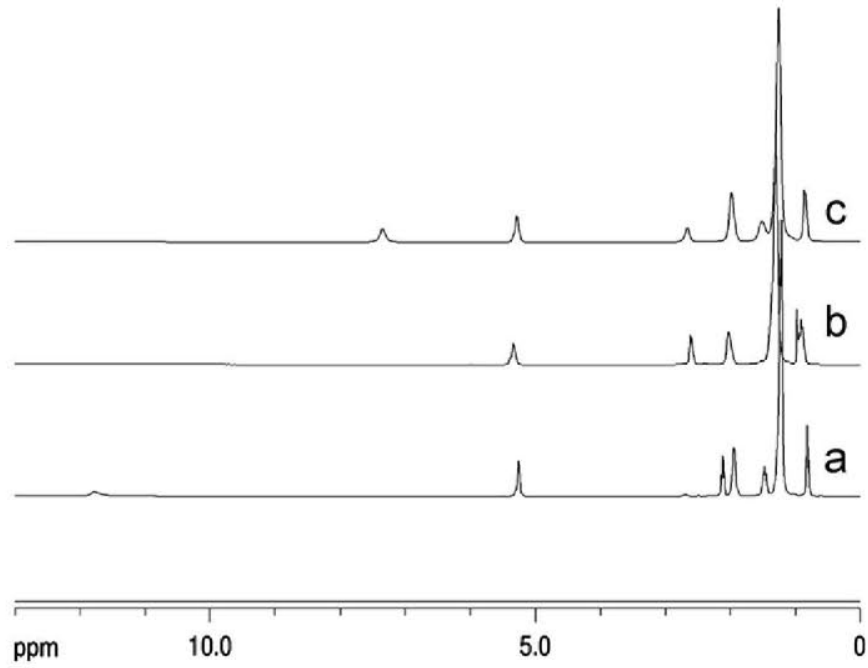

Fig. 7. ${ }^{1} \mathrm{H}$ NMR spectra in DMSO-d $\mathrm{d}_{6}$ of: (a) oleic acid (OA), (b) oleylamine (OM) and (c) dioleamide (DO).

particles could be controlled [39-41]. In our case it has been suggested that $\mathrm{OA}$ strongly binds to the $\mathrm{TiO}_{2}\{001\}$ faces $[25,28]$, whereas $\mathrm{OM}$ tends to adhere on the $\{101\}$ ones [18]. Thus, varying the proportions of these compounds it is possible to modulate the rate of hydrolysis and promote the formation of $\mathrm{TiO}_{2}$ nanoparticles with controlled shape and size [28]. Nevertheless, there is still a fact that must be considered: oleylamine and oleic acid condense exothermically in situ to form a long-chain amide, dioleamide (DO) [42], in a visible spontaneous process, which is confirmed by ${ }^{1} \mathrm{H}$ NMR spectroscopy. As shown in Fig. 7, after condensation the spectrum of the obtained product (DO) shows a new signal at $7.35 \mathrm{ppm}$, which corresponds to the $\mathrm{H}_{\mathrm{e}}$ and $\mathrm{NH}$ group. Furthermore, the lack of any signal above $10 \mathrm{ppm}$ confirms the loss of the hydrogen belonging to the carboxylic group. Finally, some signals are shifted compared to the free precursors. These a

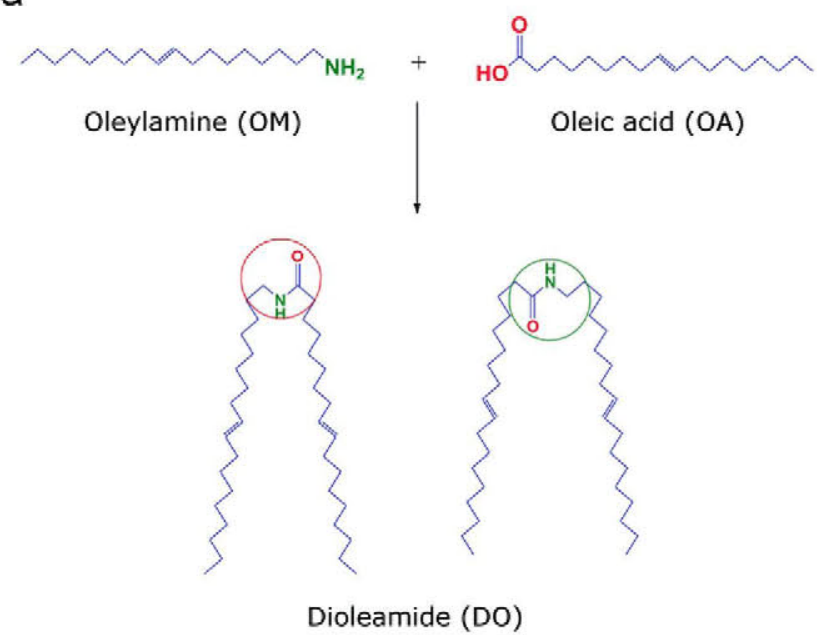

$\mathrm{b}$
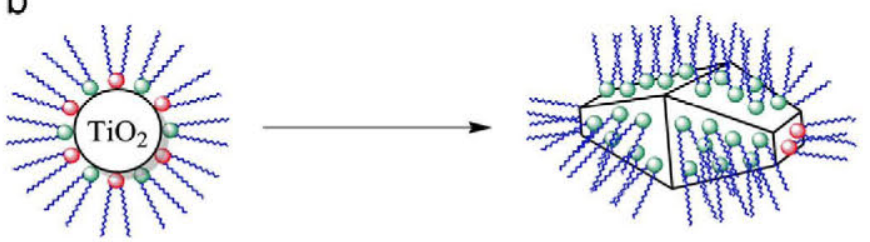

Fig. 8. (a) Oleic acid (OA) and oleylamine (OM) condensation and (b) shape control of dioleamide (DO) depending of the bond selectivity and strength.

facts confirm that the formation of DO was near completion after the addition of both compounds. This new formed molecule also plays a role as a surfactant [43], and according to our results it may also bind onto the $\mathrm{TiO}_{2}$ faces with different strengths: more selectively to the $\{001\}$ and $\{010\}$ faces through the $-\mathrm{C}=\mathrm{O}$ group and in a weaker way to the $\{101\}$ faces through the $-\mathrm{NH}$ group (Fig. 8). These selective bindings of the DO molecule to different facets of the $\mathrm{TiO}_{2}$ crystals restrict growth in the corresponding directions, as we have observed. On the 

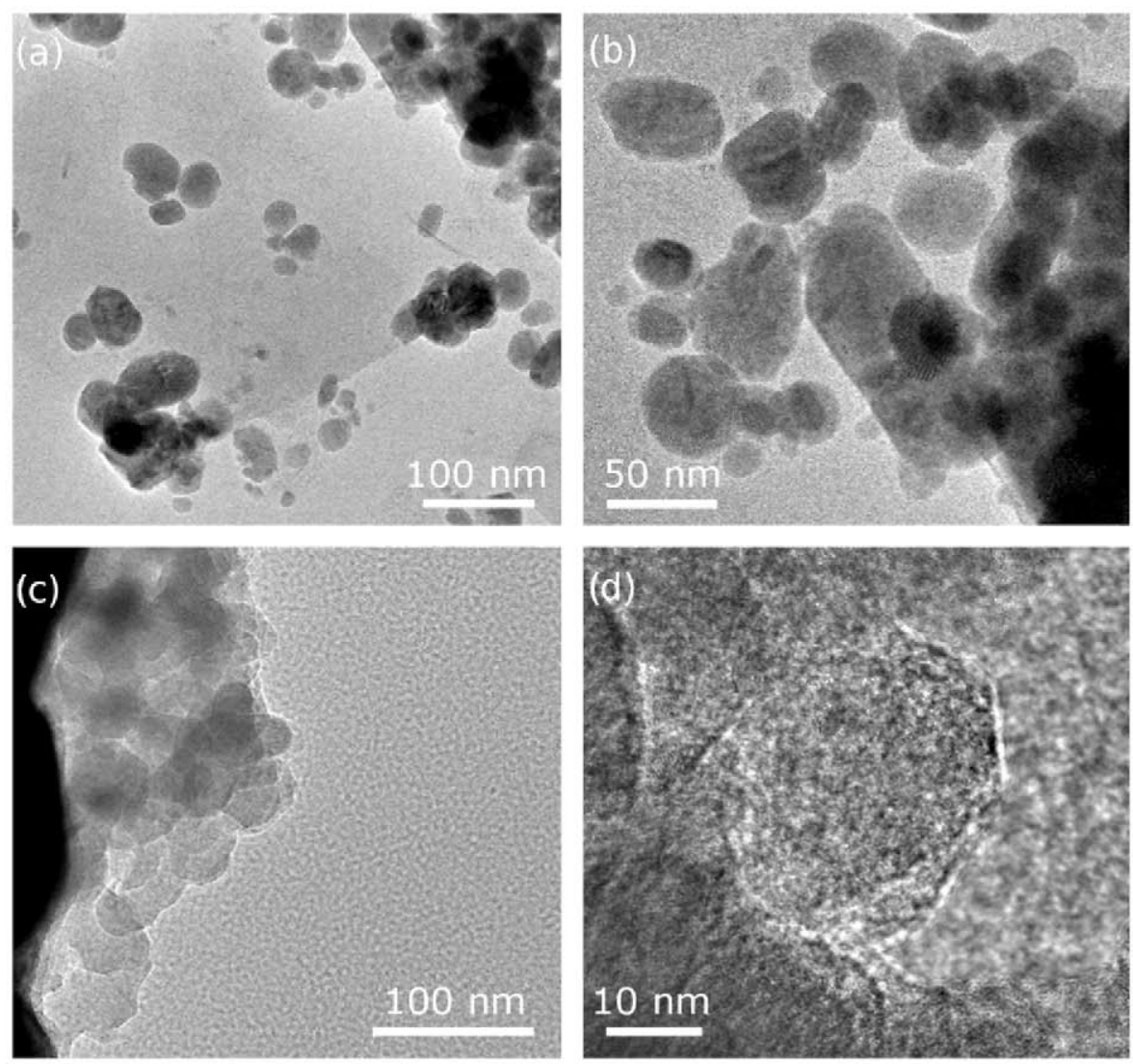

Fig. 9. TEM images of: (a) and (b) TiNP5 obtained at $78^{\circ} \mathrm{C}$ for $72 \mathrm{~h}$, (c)

c) TiNP6 and (d) an individual particle of TiNP6 obtained at $78^{\circ} \mathrm{C}$ for $72 \mathrm{~h}$.

other hand, when the $\mathrm{OA} / \mathrm{OM}$ mole ratio is changed to $6: 4$ (TiNP6 samples), there is an excess of unreacted OA and therefore an equilibrium is set between the binding of DO and $\mathrm{OA}$ to $\{001\}$ faces. Due to the higher electronegativity of the carboxylic group belonging to $\mathrm{OA}$, this probably binds more strongly and selectively to $\{001\}$ faces, and as a result both $\mathrm{TiO}_{2}$ truncated nanooctahedrons and $\mathrm{TiO}_{2}$ nanorods are promoted.

\subsection{Effect of reaction time}

In order to evaluate how reaction time affects morphology of the particles, the reactions with $5: 5$ and $6: 4$ surfactants ratios were carried out, increasing the time up to $72 \mathrm{~h}\left(T=78^{\circ} \mathrm{C}\right)$. As it can be observed in Fig. 9, the truncated rhombic nanoparticles retain their morphology, but no nanorods are observed this time. Once more crystallinity of the particles was verified by SAED (not depicted here). In the particular case of samples with 5:5 $\mathrm{OA} / \mathrm{OM}$ molar ratio, merely an increase in the average particle size $(>50 \mathrm{~nm})$ is produced. However, in samples with $6: 4 \mathrm{OA} / \mathrm{OM}$ molar ratio significant changes have been generated: the truncated octahedrons have been elongated in the [001] direction and this is due to the developing of new $\{010\}$ facets (Fig. 9(c) and (d)). This situation can be ascribed to a kinetic regime [44], i.e. to the fact that the crystals grow faster on the faces with higher surface energy: $\{001\}$ and $\{010\}$. Actually, our experiments with
6:4 OA/OM molar ratio (TiNP6 samples) suggest that the growth of the $\{001\}$ faces is initially faster than that of the $\{010\}$ ones, but beyond a certain point which we achieve by increasing the reaction time, the preferential growth switches to the $\{010\}$ faces. Furthermore, Barnard et al. [45] also found that the formation of these new $\{010\}$ facets is encouraged by conditions in which oxygenated surfaces are favored, something that we have in the TiNP6 samples with a higher amount of OA (6:4 M ratio) in the reaction medium.

\section{Conclusions}

A sustainable and versatile synthesis method has been developed which allows to obtain morphologically-tailored $\mathrm{TiO}_{2}$ nanoparticles at extraordinarily soft solution conditions. Anatase nanostructured hierarchical spheres as well as individual nanoparticles with exposed high-energy facets ( $\{001\}$ and $\{010\})$ can be obtained at atmospheric pressure and temperatures as low as $78^{\circ} \mathrm{C}$. The strategy is based on the use of a non-aqueous alcoholic reaction medium with water traces to accelerate the hydrolysis of the Ti precursor. These water traces are incorporated either from the air (atmospheric water) or from the ethanol-water azeotropic mixture (ethanol 96\%). Besides, organic surfactants based on a combination of oleic acid, oleylamine and the in situ formed dioleamide, have been employed to cap the growth in one or more crystal faces. Consequently the 
particles develop only in certain directions, and hence tailoring their morphology. The absence of fluorine-based compounds, which are typically used as capping agents to control the morphology of the growing $\mathrm{TiO}_{2}$ particles, makes the method here proposed a promising environmental-friendly alternative to current synthesis routes.

\section{Acknowledgments}

This work was supported by the Spanish Ministry of Science and Innovation (MICINN) through the projects IPT-120000-2010-033 (GESHTOS), IPT-2011-1113-310000 (NANOBAC) and CICYTMAT 2010-16614. The Spanish National Research Council (CSIC) and European Science Foundation (ESF) are also acknowledged for a $J A E-D o c$ contract to Dr. Teresa Jardiel.

\section{References}

[1] S. Liu, J. Yu, M. Jaroniec, Anatase $\mathrm{TiO}_{2}$ with dominant high-energy $\{001\}$ facets: synthesis, properties, and applications, Chemistry of Materials 23 (2011) 4085-4093.

[2] A. Hegazy, E. Prouzet, Room temperature synthesis and thermal evolution of porous nanocrystalline $\mathrm{TiO}_{2}$ anatase, Chemistry of Materials 24 (2012) 245-254.

[3] J.J. Wu, X.J. Lü, L.L. Zhang, F.Q. Huang, F.F. Xu, Dielectric constant controlled solvothermal synthesis of a $\mathrm{TiO}_{2}$ photocatalyst with tunable crystallinity: a strategy for solvent selection, European Journal of Inorganic Chemistry 19 (2009) 2789-2795.

[4] M. Li, Z.L. Hong, Y.N. Fang, F.Q. Huang, Synergistic effect of two surface complexes in enhancing visible-light photocatalytic activity of titanium dioxide, Materials Research Bulletin 43 (2008) 2179-2186.

[5] X.J. Lü, X. Mou, J.J. Wu, D.W. Zhang, L.L. Zhang, F.Q. Huang, F.F. Xu, S.M. Huang, Improved-performance dye-sensitized solar cells using $\mathrm{Nb}$-doped $\mathrm{TiO}_{2}$ electrodes: efficient electron injection and transfer, Advanced Functional Materials 20 (2010) 509-515.

[6] M. Grätzel, Photochemical cells, Nature 414 (2001) 338-344.

[7] X. Chen, S.S. Mao, Titanium dioxide nanomaterials: synthesis, properties, modifications, and applications, Chemical Reviews 107 (2007) 2891-2959.

[8] E.A. Rozhkova, I. Ulasov, B. Lai, N.M. Dimitrijevic, M.S. Lesniak, T. Rajh, A high-performance nanobio photocatalyst for targeted brain cancer therapy, Nano Letters 9 (2009) 3337-3342.

[9] D. Wang, D. Choi, J. Li, Z. Yang, Z. Nie, R. Kou, D. Hu, C. Wang, L.V. Saraf, J. Zhang, I.A. Aksay, J. Liu, Self-assembled $\mathrm{TiO}_{2}$ graphene hybrid nanostructures for enhanced Li-ion insertion, ACS Nano 3 (2009) 907-914.

[10] X.J. Lü, F.Q. Huang, X.L. Mou, Y.M. Wang, F.F. Xu, A general preparation strategy for hybrid $\mathrm{TiO}_{2}$ hierarchical spheres and their enhanced solar energy utilization efficiency, Advanced Materials 22 (2010) 3719-3722.

[11] D. Kim, A. Ghicov, S.P. Albu, P.J. Schmuki, Bamboo-type $\mathrm{TiO}_{2}$ nanotubes: improved conversion efficiency in dye-sensitized solar cells, Journal of the American Chemical Society 130 (2008) 16454-16455.

[12] S.W. Kim, T.H. Han, J. Kim, H. Gwon, H.S. Moon, S.W. Kang, S.O. Kim, K. Kang, Fabrication and electrochemical characterization of $\mathrm{TiO}_{2}$ three-dimensional nanonetwork based on peptide assembly, ACS Nano 3 (2009) 1085-1090.

[13] S. Joo, I. Muto, N. Hara, Hydrogen gas sensor using Pt- and Pdadded anodic $\mathrm{TiO}_{2}$ nanotube films, Journal of the Electrochemical Society 157 (2010) J221-J226.
[14] H.G. Yang, C.H. Sun, S.Z. Qiao, J. Zou, G. Liu, S.C. Smith, H.M. Cheng, G.Q. Lu, Anatase $\mathrm{TiO}_{2}$ single crystals with a large percentage of reactive facets, Nature 453 (2008) 638-641.

[15] H. Yang, G. Liu, S. Qiao, C. Sun, Y. Jin, S.C. Smith, J. Zou, H.M. Cheng, G.Q. Lu, Solvothermal synthesis and photoreactivity of anatase $\mathrm{TiO}_{2}$ nanosheets with dominant $\{001\}$ facets, Journal of the American Chemical Society 131 (2009) 4078-4083.

[16] X. Han, Q. Kuang, M. Jin, Z. Xie, L. Zheng, Synthesis of titania nanosheets with a high percentage of exposed (001) facets and related photocatalytic properties, Journal of the American Chemical Society 131 (2009) 3152-3153.

[17] Y. Dai, C.M. Cobley, J. Zeng, Y. Sun, Y. Xia, Synthesis of anatase $\mathrm{TiO}_{2}$ nanocrystals with exposed $\{001\}$ facets, Nano Letters 9 (2009) $2455-2459$

[18] J. Joo, S.G. Kwon, T. Yu, M. Cho, J. Lee, J. Yoon, T. Hyeon, Large-scale synthesis of $\mathrm{TiO}_{2}$ nanorods via nonhydrolytic sol-gel ester elimination reaction and their application to photocatalytic inactivation of E. coli, Journal of Physical Chemistry B 109 (2005) $15297-15302$.

[19] J. Li, L.W. Wang, Shape effects on electronic states of nanocrystals, Nano Letters 3 (2003) 1357-1363.

[20] X. Lü, S. Ding, Y. Xie, F. Huang, Non-aqueous preparation of highcrystallinity hierarchical $\mathrm{TiO}_{2}$ hollow spheres with excellent photocatalytic efficiency, European Journal of Inorganic Chemistry (2011) 2879-2883.

[21] Z. Zheng, B. Huang, X. Qin, X. Zhang, Y. Dai, M. Jiang, P. Wang, M.H. Whangbo, Highly efficient photocatalyst: $\mathrm{TiO}_{2}$ microspheres produced from $\mathrm{TiO}_{2}$ nanosheets with a high percentage of reactive \{001\} facets, Chemistry-A European Journal 15 (2009) $12576-12579$.

[22] Z. Zheng, B. Huang, J. Lu, X. Qin, X. Zhang, Y. Dai, Hierarchical $\mathrm{TiO}_{2}$ microspheres: synergetic effect of $\{001\}$ and $\{101\}$ facets for enhanced photocatalytic activity, Chemistry - A European Journal 17 (2011) 15032-15038.

[23] J.N. Hay, H.M. Raval, Synthesis of organic-inorganic hybrids via the non-hydrolytic sol-gel process, Chemistry of Materials 13 (2001) 3396-3403.

[24] M. Niederberger, M.H. Bartl, G.D. Stucky, Benzyl alcohol and transition metal chlorides as a versatile reaction system for the nonaqueous and low-temperature synthesis of crystalline nanoobjects with controlled dimensionality, Journal of the American Chemical Society 124 (2002) 13642-13643.

[25] Y.W. Jun, M.F. Casula, J.H. Sim, S.Y. Kim, J. Cheon, A.P. Alivisatos, Surfactant-assisted elimination of a high energy facet as a means of controlling the shapes of $\mathrm{TiO}_{2}$ nanocrystals, Journal of the American Chemical Society 125 (2003) 15981-15985.

[26] Z. Zhang, X. Zhong, S. Liu, D. Li, M. Han, Aminolysis route to monodisperse titania nanorods with tunable aspect ratio, Angewandte Chemie International Edition 44 (2005) 3466-3470.

[27] R. Buonsanti, V. Grillo, E. Carlino, C. Giannini, T. Kipp, T.R. Cingolani, D. Cozzoli, Nonhydrolytic synthesis of high quality anisotropically shaped brookite $\mathrm{TiO}_{2}$ nanocrystals, Journal of the American Chemical Society 130 (2008) 11223-11233.

[28] C.T. Dinh, T.D. Nguyen, F. Kleitz, T.O. Do, Shape-controlled synthesis of highly crystalline titania nanocrystals, ACS Nano 3 (2009) 3737-3743.

[29] M. Peiteado, T. Jardiel, F. Rubio, A.C. Caballero, Multipod structures of $\mathrm{ZnO}$ hydrothermally grown in the presence of $\mathrm{Zn}_{3} \mathrm{P}_{2}$, Materials Research Bulletin 45 (2010) 1586-1592.

[30] H.G. Yang, C.H. Sun, S.Z. Qiao, J. Zou, G. Liu, S.C. Smith, H.M. Cheng, G.Q. Lu, Anatase $\mathrm{TiO}_{2}$ single crystals with a large percentage of reactive facets, Nature 453 (2008) 638-641.

[31] J. Pan, G. Liu, G.Q.M. Lu, H.M. Cheng, On the true photoreactivity order of $\{001\},\{010\}$, and $\{101\}$ facets of anatase $\mathrm{TiO}_{2}$ crystals, Angewandte Chemie International Edition 50 (2011) 2133-2137.

[32] X.H. Yang, Z. Li, C. Sun, H.G. Yang, C. Li, Hydrothermal stability of $\{001\}$ faceted anatase $\mathrm{TiO}_{2}$, Chemistry of Materials 23 (2011) $3486-3494$ 
[33] C.Z. Wen, H.B. Jiang, S.Z. Qiao, H.G. Yang, G.Q. Lu, Synthesis of high-reactive facets dominated anatase $\mathrm{TiO}_{2}$, Journal of Materials Chemistry 21 (2011) 7052-7061.

[34] Y. Yin, A.P. Alivisatos, Colloidal nanocrystals synthesis and the organic-inorganic interface, Nature 437 (2005) 664-670.

[35] S.G. Kwon, T. Hyeon, Colloidal chemical synthesis and formation kinetics of uniformly sized nanocrystals of metals, oxides, and chalcogenides, Accounts of Chemical Research 41 (2008) 1696-1709.

[36] X. Wang, Q. Peng, Y. Li, Interface-mediated growth of monodispersed nanostructures, Accounts of Chemical Research 40 (2007) 635-643.

[37] J.S. Chen, Y.L. Tan, C.M. Li, Y.L. Cheah, D. Luan, S. Madhavi, F.Y.C. Boey, L.A. Archer, X.W. Lou, Constructing hierarchical spheres from large ultrathin anatase $\mathrm{TiO}_{2}$ nanosheets with nearly $100 \%$ exposed (001) facets for fast reversible lithium storage, Journal of the American Chemical Society 132 (2010) 6124-6130.

[38] J. Livage, M. Henry, C. Sanchez, Sol-gel chemistry of transition metal oxides, Progress in Solid State Chemistry 18 (1988) 259-341.

[39] E. Ramirez, S. Jansat, K. Philippot, P. Lecante, M. Gomez, A.M. Masdeu-Bulto, B. Chaudret, Influence of organic ligands on the stabilization of palladium nanoparticles, Journal of Organometallic Chemistry 689 (2004) 4601-4610.

[40] H.X. Mai, Y.W. Zhang, R. Si, Z.G. Yan, L.D. Sun, L.P. You, C.H. Yan, High-quality sodium rare-earth fluoride nanocrystals controlled synthesis and opticals properties, Journal of the American Chemical Society 128 (2006) 6426-6436.

[41] J. Watt, N. Young, S. Haigh, A. Kirkland, R.D. Tilley, Synthesis and structural characterization of branched palladium nanostructures, Advanced Materials 21 (2009) 2288-2293.

[42] B.D. Fahlman, Nanoscale building blocks and applications, in: Materials Chemistry, 2nd ed., Springer-Verlag New York LLC, New York, 2008, pp. 517-518.

[43] H. Wu, Y. Yang, Y.C. Cao, Synthesis of colloidal uranium-dioxide nanocrystals, Journal of the American Chemical Society 128 (2006) 16522-16523.

[44] Y.W. Jun, Y.Y. Jun, J.J. Cheon, Architectural control of magnetic semiconductor nanocrystals, Journal of the American Chemical Society 124 (2001) 615-619.

[45] A.S. Barnard, L.A. Curtiss, Prediction of $\mathrm{TiO}_{2}$ nanoparticle phase and shape transitions controlled by surface chemistry, Nano Letters 5 (2005) 1261-1266. 